\title{
Kulturgeschichtliche Aspekte des Schmerzes
}

\author{
M. Reiber
}

\begin{abstract}
Schmerz ist - neben der Liebe - die fundamentale menschliche Erfahrung. Schmerz ist der eigentliche Gradmesser für menschliches Wohl- oder Missbefinden. Schmerz ist der gewichtigste Ursprung der Medizin, seine Überwindung das untrüglichste Zeichen ihres Erfolgs. Schmerz ist das universale Mittel der Gewalt; er ist Instrument der Drohung, Strafe oder Busse. Schmerz ist Natur und Schmerz ist Kultur: er wird mit dem Körper erfahren, mit der Seele erlitten, mit dem Geist gedeutet. Schmerz ist somit - wie die Medizin insgesamt - Gegenstand der Naturwissenschaft, der Kulturwissenschaft, der Geisteswissenschaft, überdies der Sozialwissenschaft, und zwar dort, wo die gesellschaftlichen und institutionellen Bedingungen von Schmerz und Krankheit ins Auge gefasst werden.

Die Mobilität und Migration in der modernen Gesellschaft ermöglicht dem Arzt von heute, die kulturwissenschaftliche Dimension seines Berufes nicht mehr nur ungefähr zu kennen. Er kann sie konkret erfahren, indem er erlebt, wie die unterschiedlichsten Welt- und Menschenbilder in sein Handeln hineinwirken. Er beobachtet die Variabilität von Beschwerden und Behandlungsformen in Abhängigkeit von Mentalitäten und Volkszugehörigkeiten. Und dabei begreift er nunmehr aus eigener Anschauung, was er schon wusste: dass das, was als krank oder gesund gilt, sich nicht als blosses naturwissenschaftliches Fakt präsentiert, sondern sich erst im Lichte der jeweiligen gesellschaftlichen Wertbegriffe erschliesst. So gesehen sind in der Medizin Natur und Kultur aufs engste miteinander verbunden - dort die Naturgesetze, hier die Ideen, Werte und Traditionen. Ich will im folgenden mit Blick auf den Schmerz und seine Behandlung an einige wenige Gesichtspunkte unserer Kulturgeschichte erinnern.
\end{abstract}

\section{Konzeptionen des Phänomens Schmerz}

In der Historiographie der Naturwissenschaft werden fünf massgebliche Konzeptionen zur Beschreibung des Phänomens Schmerz hervorgehoben [1]. Erstens, die seit der griechischen Antike bis ins 19. Jahrhundert tradierte Meinung, der Schmerz sei ein Gefühl,

Korrespondenz:

Dr. med. Matthias Reiber

Leiter Psychosomatik

Rehabilitationsklinik

CH-7317 Valens das zum Lustgefühl den Gegensatz bildet. Dieses sei im Herzen zu orten. Und dieses Gefühl sei weder ein blosser Wahrnehmungseindruck noch einfache Sinnesmodalität; der Schmerz sei eine den gesamten Bewusstseinsprozess prägende affektive Erscheinung.

Dieser sogenannten Affekttheorie folgt ideengeschichtlich, zweitens, die Vorstellung, dass der Schmerz eine einfache Empfindung darstelle, die in einem spezifischen System übermittelt und verarbeitet werde. Das morphologische Substrat dieses physiologischen Systems seien schmerzspezifische Rezeptoren, Schmerzleitungen und eigene Schmerzzentren im Gehirn.

Neben dieser sogenannten Spezifitätstheorie und der vorgenannten Affekttheorie besteht, drittens, die Auffassung, Schmerz sei die Wahrnehmung einer Körperstörung oder Körperverletzung und insofern eine sinnvolle biologische Einrichtung. Der Begriff der Schmerzwahrnehmung profiliert diese sogenannte Wahrnehmungstheorie gegenüber der Affekttheorie, die den psychischen Prozess des Fühlens favorisiert, und der den physischen Vorgang des Empfindens betonenden Spezifitätstheorie als ein Konzept, das gewissermassen ohne die subjektive Disposition des Fühlenden oder Empfindenden auskommt und ganz das wahrnehmende Bewusstsein objektiver körperlicher Pathologien ins Auge fasst.

Eine vierte Auffassung über den Schmerz etablierte sich seit den sechziger Jahren des 20. Jahrhunderts unter dem Namen funktionalistische Schmerztheorie. Diese Theorie fokussiert die Ansicht, dass Schmerz das Resultat eines multidimensionalen Gefüges kognitiver, affektiver, motivationaler und verhaltensmässiger Elemente sei. Schmerz sei demzufolge nicht einfach Gefühl, Empfindung oder Wahrnehmung, sondern ein komplexer Zustand, der einer funktionalen Zerlegung, einer eigentlichen Analyse, zugänglich sei.

Kennzeichnend für eine fünfte Position ist schliesslich die behavioristische Überzeugung, dass Schmerz Schmerzverhalten sei, das heisst: die Beschreibung von Schmerz könne ausschliesslich mit den Begriffen objektiven Verhaltens erfolgen.

Jede der fünf Ansichten weist Stärken auf. Die erste, die Affekttheorie, besticht dadurch, dass sie den - im Vergleich zu den übrigen Empfindungen - herausragenden Einfluss des Schmerzes auf das Bewusstsein betont: denn in der Tat bewegt uns der Schmerz auf ganz besondere Weise. Es ist insofern treffend, ihn eine Emotion zu nennen. Die zweite, die Spezifitätstheorie, gewinnt ihre Anziehungskraft daraus, dass sie mit einem psychophysiologischen Modell auf die leib-seelische Einheit hinweist: Der Schmerzapparat spannt sich gewissermassen zwischen Haut und Hirn aus und verbindet so Leib und Seele. Die Wahrnehmungstheorie, drittens, die den Schmerz als Boten einer Körperstörung interpretiert, gefällt schon dadurch, dass sie dem Schmerz einen Sinn, einen immerhin biologischen Sinn zuschreibt (wenngleich wir natürlich alle einwenden müssen, dass bei chronifizierten Schmerzen, von denen beim heutigen Symposium die Rede ist, dieser erkennbare Sinn ver- 
loren ist, indem der Schmerz nicht mehr Symptom ist, sondern biologisch sinnentleertes Syndrom). Und selbstverständlich kann die mit den Trendbegriffen Multidimensionalität und Komplexität argumentierende vierte Schmerztheorie, die sämtliche Aspekte im psychologischen Wirkungsgefüge kennt, viel $\mathrm{Zu}$ stimmung ernten. Auch der ziemlich radikal daherkommenden fünften, der behavioristischen Anschauung lässt sich etwas abgewinnen, weil sie den Blick dafür schärft, dass letztlich das Verhalten das Mass aller Dinge ist - in aller Psychotherapie, wie ich meine, und so auch in der psychotherapeutisch unterstützten Schmerzbewältigung.

Die Schwächen dieser Betrachtungsweisen liegen allgemein in ihrem Reduktionismus. Sie betonen wesentliche Gesichtspunkte und erweisen sich zur Erklärung von Teilaspekten als nützlich. Ihr Nutzen ist aber begrenzt; ihre Aussagekraft richtet sich lediglich auf einzelne Anwendungsbereiche. In Anbetracht ihrer explanatorischen Schwächen muss es nun darauf ankommen, die Stärken der einzelnen Theorien sinnvoll zu nutzen, anstatt sie ihrer Schwächen wegen in toto zu verwerfen. Das bedeutet, sich die intellektuelle Freiheit zu nehmen, das Denkgebäude zu wechseln, wo dies für die Praxis sinnvoll ist. Es geht nicht um die Suche nach der einzelnen allgemeingültigen Theorie über den Schmerz. Es geht vielmehr um die Anerkenntnis, dass wir uns bei einer Annäherung an das Mysterium Schmerz [2] mit Gewinn in einem Netzwerk von Modellen bewegen, wenn unser Wissen in der therapeutischen Praxis nützen soll.

\section{Vernetztes Denken über den Schmerz}

Ich rede also vom vernetzten Denken über den Schmerz. Dieses ist, wie bereits gesagt, erstens, dadurch zu charakterisieren, dass wir uns in einem Netz von Modellen möglichst frei bewegen, dabei nach dem für unseren Zweck brauchbarsten Modell Ausschau haltend. Dieses Denken ist, zweitens, durch eine Sichtweise geprägt, die zirkulär genannt wird. Das heisst: die Abläufe, um die es geht, sind, dieser Auffassung gemäss, kreisförmig miteinander verbunden. Jede Aktion hat demnach nicht nur Auswirkungen auf andere Faktoren, sondern auch auf den agierenden Faktor selbst. Dieses Denken ist, drittens, in dem Sinn ganzheitlich, als es den Teilaspekt immer in seiner Bedeutung für das Gesamte zu verstehen sucht wie umgekehrt das Gesamte in seiner Bedeutung für den Teil, um auf diesem Weg schliesslich das Ganze als etwas anzusehen, was mehr und zugleich anders ist als nur die Summe der Teile.

Somit rede ich also auch von einer systemischen Betrachtungsweise. Kommunikation ist der zentrale Begriff systemischen Denkens. Unter dem Gesichtspunkt der Kommunikation erscheint Schmerz als Kommunikationsmittel mit der Möglichkeit der Kommunikationsstörung dort, wo das durch ihn Mitgeteilte nicht verstanden wird.

Das Paradigma des systemischen Denkens über den Schmerz impliziert nicht nur ein zweckmässiges
Verknüpfen von Anschauungen und Modellen eines Fachgebiets. Das Netz von Ansichten, Theorien und Erfahrungen durchdringt das weite Feld des Phänomens Schmerz fächerübergreifend. Den fächerübergreifenden Diskurs führen neben Vertretern der Naturwissenschaft auch solche der Geisteswissenschaft, neben Repräsentanten der theoretischen Medizin auch Literaturwissenschafter, Philosophen, Theologen und - im Schnittpunkt der Sparten - einige Psychiater.

Es geht um eines der grossen Themen der Menschheit, ein Thema, das keinem Menschen wirklich erst nahegebracht werden muss, über das Wissenschafter niemals allein aus dem Blickwinkel ihrer Disziplinen, sondern immer auch aus einer eigenen, persönlichen Sicht zu berichten haben. Der schmerzgeplagte Mensch, der Mensch in seiner Not und Bedürftigkeit: die Auseinandersetzung damit war im übrigen nie nur ein Anliegen der Wissenschaft, sondern immer auch der Künste.

Warum dieser Ruf nach einem vernetzten Denken über den Schmerz, nach einem fächerübergreifenden Diskurs, ja sogar nach einem Dialog der Wissenschafter mit den Kunstschaffenden? Die Antworten sind einfach: Der Aufruf zum vernetzten Denken ist die Folge des Erkennens, dass sich das lineare, das monokausale Denken als Grundlage der Schmerzbehandlung sehr oft nicht bewährt. Der Ruf nach einem umfassenden, offenen Diskurs gleicht hierbei einem Hilferuf: Wir Wissenschafter und Heilkundigen des Schmerzes sind, auf uns selbst gestellt, überfordert. Wir brauchen nicht nur den Rat des jeweils anderen medizinischen Spezialisten. Wir brauchen das Denken derer, denen Sinn- und Wertfragen zu denken geben. Denn der Schmerz wird dort, wo er nicht vorübergeht und trotz aller Behandlung überdauert, ein menschliches Leben zeichnen. Er wird Zukunftspläne durchkreuzen, Hoffnungen zunichte machen; er wird Güter, auf die sich das Leben ausgerichtet hat, nehmen oder in unerreichbare Ferne rücken und also Sinn- und Wertfragen aufwerfen, Fragen, die zum Umdenken und Umorientieren herausfordern. Auf diesem Terrain trägt der Boden der naturwissenschaftlich-technischen Medizin nicht mehr. Und da die Not gleichwohl Handeln verlangt, ist es gut, das, was durch die Begrenzungen dieser Medizin bricht, aufzufangen. Das Krankengut wird gleichsam im Notlazarett der Psychosomatik empfangen. Psychosomatische Medizin ist ein Notbehelf - wie übrigens der Begriff der Psychosomatik selbst, der unglücklicherweise den Determinismus psychische Ursache: somatische Folgen suggeriert und somit einem linearen, monokausalen Denken Vorschub leistet, gegen das sich diese Psychosomatik, verstanden als eine Heilkunst, welche die Vernetzung der vielfältigen körperlichen, seelischen und sozialen Bezüge studiert, gerade wendet.

Im Namen einer Heilkunde für den ganzen Menschen ist in der Kulturgeschichte auch in früheren Epochen die Koalition der Medizin mit der Philosophie, der Literatur, mit der Theologie und den Künsten verwirklicht worden: Gesundheit, Krankheit und Schmerz 
wurden in der antiken Heilkunst, der Keimzelle unserer wissenschaftlichen Medizin, vor dem Hintergrund eines kosmologisch-anthropologischen Weltbildes interpretiert. Die Natur im allgemeinen und die menschliche Natur standen dieser Auffassung gemäss in steter Wechselbeziehung; es wurde eine eigentliche Parallelisierung des Makrokosmos Natur und des Mikrokosmos Mensch angenommen. Gesundheit, Krankheit und Schmerz wurden in einem Zusammenhang mit der Natur, beispielsweise mit den Jahreszeiten, mit dem Klima und der Landschaft gebracht. Gesundheit wurde als Zustand der Harmonie oder Balance in diesem Wirkungsgefüge von Elementen, Qualitäten, Säften, Organen, Temperamenten und Zeiten verstanden, Krankheit als Dysbalance - und Schmerz im besonderen als Leiden an der Unvollkommenheit der Natur, an der Schadhaftigkeit der Welt. Die Medizin des christlichen Mittelalters deutete Gesundheit, Krankheit und Schmerz theologisch. Der leidende Christus und der das Leiden überwindende Heiland waren die Leitfiguren für den Kranken und dessen Heiler. Krankheit und Schmerz wurden als Folgen des Sündenfalls angesehen, als Ausdruck der Besessenheit; sie bedeuteten Prüfungen durch Gott und verwiesen - aber niemals allein - auf individuelle Schuld. Da sich göttliches Wirken in der Natur offenbart, stand Schmerz - nicht anders als in antiker Weltsicht - für das Leiden an den Trennungen von der Natur.

\section{Cartesianische Umdeutung des Schmerzes}

Bis tief ins 17. Jahrhundert hinein prägte im wesentlichen diese Auffassung ärztliches Denken. Dann allerdings trat ein Wandel ein, dessen Konsequenzen bis heute den Diskurs über den Schmerz leiten [3]. René Descartes (1596-1650) etablierte um 1640 die Überzeugung, dass die Seele eine vom Körper verschiedene Substanz sei. Das Bewusstsein, das heisst das erkennende Subjekt, sei von der Gegenstandswelt, das heisst von der Körperwelt, getrennt. Der Leib wurde damit in der Vorstellung zum entseelten Körper, zum Apparat, der unter technischen Gesichtspunkten betrachtet werden konnte. Die getrennte Betrachtung von Körper und Seele bedeutete nun in der Anwendung auf das Schmerzproblem, dass dem Schmerz sowohl ein körperlicher als auch ein seelischer Bereich je eigener Ordnung zugesprochen wurde. Damit wurde auch erstmals die Frage nach den körperlichen Bedingungen des Schmerzes gestellt. Für den Schmerz tat sich die Welt des Körpers auf. In der Körperwelt wurde er in seiner Entstehung und Funktion analysiert. Und je besser man seine Funktionsweise begriff, desto besser glaubte man seiner Herr werden zu können. Die Schmerzbeseitigung wurde zu einem zentralen Anliegen. Darin nun zeigte sich die eigentliche cartesianische Umdeutung des Schmerzes: die Schmerzbeseitigung trat an die Stelle der Schmerzbewältigung. Wurde der Schmerz traditionell als ein unaufhebbares Grundübel der Natur angesehen, ein Wesensmerkmal der Welt wie Licht, Luft und Wasser, eine Gegebenheit, der sich der be- troffene Mensch stellen musste, die er zu erdulden und zu bewältigen hatte, so brachte der Rationalismus jetzt die Einstellung, dass sich der Mensch dem Schmerz nicht weiter ausliefern müsse.

Die Erfolge der modernen somatischen Schmerztherapie sind ohne den konsequenten Gedanken, dass Schmerz als integraler Bestandteil der Körperwelt mit den Mitteln der Körperwelt beseitigt werden kann und auch soll, nicht vorstellbar. Die rationalistische Trennung von Körper und Seele bedeutete für das Denken über den Schmerz aber auch eine Trennung von Schmerz und Sinn. Dem kollektiv erfahrbaren übergeordneten Sinn früherer Zeiten, dem Sinn des Schmerzes als ein unabänderliches Zeichen der widergöttlichen, der disharmonischen, der unvollkommenen Natur trat nun die subjektiv erlebte Sinnlosigkeit eines im ungünstigsten Fall unüberwindbaren Schmerzes gegenüber, die sinnlose Qual angesichts eines Schmerzes, der nur als ein lästiges, ein vermeidbares Übel angesehen wurde.

Wie gesagt: Der cartesianische Rationalismus ist aus ärztlicher Sicht nicht zu beklagen. Er ist die Grundlage der unbestreitbaren Erfolge der Schulmedizin - auch in der Schmerztherapie. Aber wir sollten uns gegen die einseitigen naturwissenschaftlichen Entwicklungen stellen, die Folgen dieser rationalistischen Weltsicht sind. Unsere Einwände sind dabei nicht neu. Die hochgespannten Erwartungen, die Descartes und seine Schüler in das mathematischmechanische Denken setzten, riefen seit dem Ende des 17. Jahrhunderts ärztliche Gegenbewegungen hervor. Die Psychovitalisten unter ihrem Vordenker Georg Ernst Stahl (1659-1734) markierten hier den Gegenpol zu Descartes, indem sie sich nicht nur zur herkömmlichen Auffassung von der Leib-Seele-Einheit bekannten, sondern sogar von einem Primat der Seele ausgingen. Zwischen diesen Extrempositionen entfachte sich im 18. Jahrhundert unter gelehrten Ärzten eine äusserst lebhaft geführte Diskussion über die Wechselbeziehungen zwischen Körper und Seele [4]. Viele fühlten sich herausgefordert, der cartesianischen Behauptung, Körper und Seele existierten getrennt, ein Modell entgegenzusetzen, das ihre ärztliche Erfahrung erklären würde, wonach der Körper und die Seele fortlaufend ineinanderwirken müssten. Man nannte diese Ärzte damals philosophische Ärzte. Sie verlangten - im Gegenzug zum rationalistischen Reduktionismus - den interdisziplinären Diskurs. Ihre Disziplin nannten sie - mit den Worten Johann August Unzers (1727-1799) aus dem Jahr 1746 - eine "mitlere Wissenschaft", die "zwischen der Weltweisheit und der Arzneiwissenschaft zu stehen kommen müste» [5].

Damit knüpften die philosophischen Ärzte des 18. Jahrhunderts bewusst an alte ärztliche Traditionen an. Denn seit dem Altertum war es im Grunde selbstverständlich, dass die Sorge für den Körper dem Arzt unausweichlich auch die Sorge für die Seele gebot. Diese Konstante ärztlichen Denkens, die sich gelegentlich gegen den Anspruch der Philosophie zu behaupten hatte, die Seele sei allein ihr Feld, war seit der Antike untrennbar mit der Diätetik verbunden [6]. 


\section{Diätetik und Schmerzmedizin}

Was ist Diätetik? Diätetik ist nicht Diät. Es ist nicht oder jedenfalls weitaus mehr als Haferschleim und Kamillentee. Diätetik ist nichts weniger als die umfassende Lehre von der richtigen, der guten, der gesunden Lebensweise. Systematische Diätetik ist eine Errungenschaft der antiken Medizin. Sie war eines und dabei das vorrangige der drei therapeutischen Prinzipien Diätetik, Arzneibehandlung und chirurgischer Eingriff. In dieser Rangfolge wurden die Behandlungen geplant. Es war Aufgabe des Arztes, zuerst die diätetischen Mittel auszuschöpfen, bevor er Arzneien heranzog; halfen auch diese nicht, durfte er zum Messer greifen [7]. Bis um die Mitte des 19. Jahrhunderts folgten die Ärzte diesem traditionellen Dreischritt der antiken Diätetik. Dann verlor die Diätetik unter der stürmischen Entfaltung der naturwissenschaftlichen Medizin ihren Vorrang. Der therapeutische Schwerpunkt verlagerte sich zur medikamentösen und chirurgischen Behandlung.

Medizin als Diätetik zu betreiben, bedeutete vorerst, die Lebensweise in sechs verschiedenen Bereichen zu untersuchen und allenfalls zu regeln: Erstens Licht und Luft, zweitens Speis' und Trank, drittens körperliche Bewegung und Ruhe, viertens Schlafen und Wachen, fünftens Leerung und Füllung des Körpers, sechstens Bewegung des Gemüts. So gesehen zielt Diätetik auf genau das, worauf auch heute unsere ärztliche Aufmerksamkeit fällt, wenn wir unseren Horizont weiten, das vernetzte Denken kultivieren, um also, so gut es geht, an den ganzen Menschen zu gelangen.

Die Wurzeln der heutigen Präventivmedizin in der antiken Diätetik sind besonders augenscheinlich. Aber auch die psychosomatischen Bestrebungen des 20. Jahrhunderts sind im Kern diätetisches Denken. Vor allem aber - und das ist der eigentliche Grund, dass ich an den Begriff der Diätetik erinnere: Rehabilitationsmedizin ist diätetische Medizin im ursprünglichen Sinn. Es ist eine Medizin, die den Mikrokosmos Mensch konsequent auf den Makrokosmos Welt bezieht. Es ist eine Medizin, die sich nicht einfach als Instrument dem Menschen überlässt; vielmehr will sie ihn selbst vor die Aufgabe stellen, sein Leben in die Hand zu nehmen. Es ist eine Medizin, die der körperlichen und der seelischen Ordnung gleichermassen Aufmerksamkeit schenkt. Es ist eine Medizin, die dem Individuum die je besonderen Anlagen und Umstände aufzeigen will, damit diesen Rechnung getragen werde und die Balance, das Masshalten des $\mathrm{Zu}-$ träglichen auf Dauer gelinge. Es ist eine Medizin, die menschliches Wohlverhalten lehrt, damit das Leben glücke. Es ist eine Medizin, die hierdurch eines der grossen Themen der abendländischen Philosophie aufnimmt, die Frage, wie man gut und richtig lebt, wie man leben soll, damit sich Harmonie einstellt, nach innen und nach aussen.

In einer Rehabilitationsmedizin des Schmerzes, die an die Tradition der Diätetik anknüpft, lassen sich Ansichten über Natur und Kultur so verbinden, dass sich eine Perspektive auf die doppelte Natur des Menschen auftut, auf den Menschen in seinem leibseelischen und geistigen Wesen.

\section{Literatur}

1 Grahek N. Schmerz (Naturwissenschaft und Medizin). In: Ritter J, Gründer K (Hrsg.). Historisches Wörterbuch der Philosophie. Basel: Schwabe \& Co; 1992. Bd. 8. S. 1324-30.

2 Brena SF. The mystery of pain: Is pain a sensation? Management of patients with chronic pain. New York: Spectrum Publications; 1982

3 Toellner R. Die Umbewertung des Schmerzes im 17. Jahrhundert in ihren Voraussetzungen und Folgen. Medizinhist J 1971;1:36-44.

4 Zelle C (Hrsg.). Vernünftige Ärzte. Hallesche Psychomediziner und die Anfänge der Anthropologie in der deutschsprachigen Frühaufklärung. Arbeitsgespräch am Interdisziplinären Zentrum für die Erforschung der Europäischen Aufklärung IZEA in Halle vom 8. bis 10. Oktober 1997.

(Hallesche Beiträge zur Europäischen Aufklärung. Bd. 19). Tübingen: Niemeyer; 2001 (in Vorbereitung).

5 Reiber M. Anatomie eines Bestsellers. Johann August Unzers Wochenschrift «Der Arzt» (1759-1764). (Das achtzehnte Jahrhundert. Suppl. 8). Göttingen: Wallstein; 1999. S. 23.

6 Edelstein L. Antike Diätetik. Die Antike 1931;7:255-70. (Neudruck in: Medizinhist J 1966;1:162-74).

7 Bock AW. Diätetische Wundbehandlung im Mittelalter. Kyklos. Jahrbuch für Geschichte und Philosophie der Medizin 1929;2:258-71. 